\title{
Field and laboratory preparedness: challenges to rolling out new multidrug- resistant tuberculosis diagnostics
}

\author{
Anne M. J. Griffin, ${ }^{1,2}$ Luz Caviedes, ${ }^{3}$ Robert Gilman, ${ }^{3,4,5}$ \\ Jorge Coronel, ${ }^{3}$ Freddy Delgado, ${ }^{6}$ MaryLuz Quispe, ${ }^{6}$ \\ and David A. J. Moore $3,4,5$
}

Suggested citation

Griffin AMJ, Caviedes L, Gilman R, Coronel J, Delgado F, Quispe ML, et al. Field and laboratory preparedness: challenges to rolling out new multidrug-resistant tuberculosis diagnostics. Rev Panam Salud Publica. 2009;26(2):120-7.

ABSTRACT Objectives. In a pilot implementation project of the microscopic-observation drugsusceptibility methodology, we conducted a process evaluation to identify health system and logistic challenges that need to be addressed in order to harness the benefits of rolling out promising new diagnostic tools for multidrug-resistant tuberculosis (MDRTB).

Methods. Regional data relating to health system practices and performance related to the MDRTB diagnostic algorithm were collected at health center, local, and regional laboratories. Results. Parallel implementation of a new test and an existing method creates demands on funds, personnel, sample transport, and information systems in addition to new test startup costs. Obviating the need for primary culture at intermediate laboratories through direct drug susceptibility testing (DST) at the regional reference laboratory significantly reduces delay. Field application of well-defined national guidelines for DST is patchy. If fidelity to national guidelines were perfect, DST requests would increase more than 50-fold, with important implications for laboratory capacity.

Conclusions. Implementing a new MDRTB diagnostic presents challenges to the laboratory environment, the existing DST process, and the application of national guidelines in peripheral clinics. Assessing each element can maximize efficient use of a new tool. Specifically, strengthening systems for transferring samples to the laboratory and delivering results to the requesting clinic in addition to investing in personnel and laboratory resources are integral to harnessing the benefits of high-performance new diagnostic tests and can bring added value to other programs in the health care system.

Key words Mycobacterium tuberculosis; tuberculosis, multidrug-resistant; health plan implementation; clinical laboratory techniques; Peru.

Massachusetts General Hospital, Medicine-Pediatrics, Boston, Massachusetts 02114, United States of America. Send correspondence and reprint requests to: Anne M. J. Griffin, Massachusetts General Hospital, Medicine-Pediatrics, 55 Fruit Street, Boston, Massachusetts 02114, United States of America; telephone: +1 617595 5398; e-mail: amjgriffin@gmail.com

2 Mount Sinai School of Medicine, One Gustave L. Levy Plaza, New York, New York 10029, United States of America.

3 Laboratorio de Investigación de Enfermedades Infecciosas, Universidad Peruana Cayetano Heredia, Lima, Perú.
The growing imperative for more widespread application of new diagnostic tests

4 Department of International Health, Johns Hopkins University, Bloomberg School of Public Health, Baltimore, Maryland, United States of America.

5 Imperial College London Wellcome Centre for Clinical Tropical Medicine, Department of Infectious Diseases and Immunity, Imperial College London, London, United Kingdom.

6 Hospital Honorio Delgado, Programa de Tuberculosos, Dirección de Salud, Arequipa, Perú. for tuberculosis (TB) and multidrugresistant tuberculosis (MDRTB) suitable for resource-poor settings (1) is well recognized. Implementation raises significant challenges where infrastructure is limited, and operational research (2) is needed to define the optimal utilization of scant resources. High-quality analytical test performance is important but is insufficient to guarantee successful 
implementation into clinical service. To fully harness the advantages of a new test, there are at least three additional key requirements: (1) it must be readily and sustainably available in the laboratory, (2) the health system must utilize the new tool effectively, and (3) the target population must have easy access.

Prospective evaluation of the implementation process can help identify and address problems, both to maximize test use in the present and to allow for improved implementation elsewhere in the future. Understanding the environment where the diagnostic test is being introduced is the first element of ongoing implementation evaluation. In resourcepoor settings, anticipating obstacles to test sustainability, especially continuing funding, should be prioritized $(3,4)$.

Two novel, low-cost diagnostic tests for rapid detection of MDRTB have been approved by the National TB program of Peru and are ready to roll out: the microscopic-observation drug-susceptibility (MODS) assay (5-10) and the Griess (11) methodology. MODS and Griess are culture-based methods in liquid and solid media, respectively, both of which significantly cut the conventional microbiological delay in detecting MDRTB, mainly because they are performed directly from sputum rather than indirectly from strains cultured from sputum. When the opportunity arose to translate MODS for the first time from the research laboratory to a clinical service laboratory in a pilot implementation, we set out to evaluate the enabling environment and precisely define the challenges of rollout already familiar to those who work in the field. Our specific objectives were to describe the necessary laboratory environment used for implementation, the existing system for drug susceptibility testing (DST), the fidelity of clinical practice in the field to national recommendations for DST, and the degree to which geographic variation in DST requesting patterns matched that predicted by geographic variation in reported risk factor prevalence.

\section{MATERIALS AND METHODS}

\section{Setting}

This study was conducted in the regional reference laboratory (biosafety level 2) of the TB program in Arequipa (population 1140810 ) and local health clinics and laboratories. Located in southern Peru, the region of Arequipa spans Andean mountain and coastal desert; the regional capital is the second largest city in Peru. Laboratory and regional TB program staff are responsible for quality control of peripheral health facility sputum-smear-based TB diagnosis, providing first-line DST, forwarding cultured strains to the national TB reference laboratory at the National Institute for Health/Instituto Nacional de Salud (INS) for second-line DST, and coordinating MDRTB treatment in 4 hospitals, 51 health centers, 182 health posts, and 38 laboratories. The 2004 incidence of smear-positive TB (12) in the region was 47 per 100000 annually (reaching 118 per 100000 annually in some districts) and MDRTB incidence was 3.6 per 100000 annually. Forty-five clinics in urban and rural areas identified by the TB control program as having high MDRTB burden were selected for study. Medical, laboratory, and communication infrastructures ranged from physician-led centers with 24-hour services, on-site TB culture, and fax and Internet to nurse-led health posts with half-day services and weekly physician visits with no laboratory and no telephone.

\section{Design and procedures}

Four elements of the implementation setting were investigated: the laboratory environment, the mechanism and flow of samples and results within the existing DST system, fidelity to national guidelines for requesting of DST, and projected and actual geographic distribution of DST requests.

Laboratory environment. The logistic and physical aspects of implementation, including laboratory technician time and training, laboratory space, and costs, were recorded. Laboratory technician time spent in MODS-related activities was prospectively recorded in a daily workhour diary, and comparative timing of each step of direct MODS DST with the existing standard (proportions method from Ogawa culture isolates) ${ }^{7}$ was measured

\footnotetext{
MODS tests susceptibility to isoniazid and rifampin, while the proportions method tests susceptibility to isoniazid, rifampin, streptomycin, and ethambutol. MODS data are combined and averaged with stopwatch-timed observations in Arequipa and two other laboratories because of the small numbers of timed observations in Arequipa.
}

with a stopwatch. Opportunity cost calculations assumed a \$455 monthly salary and a 40-hour work week. The capital and recurrent costs of the implementation package were recorded on a spreadsheet. Space used for performance of MODS and related equipment was measured.

Existing DST system. The stepwise chronology of the flow of samples and results from when a patient first has a DST solicited on his or her behalf at a health center to receipt of a DST result was retrospectively evaluated. The paper trail was traced for 139 DSTs from the Arequipa regional reference laboratory where they were performed, to the subregional laboratories that cultured the initial samples, and to the peripheral clinics that first requested the DST. Data were abstracted from five different sample request and processing registries maintained in the regional reference laboratory.

Fidelity to national DST guidelines. We assessed how national guidelines for soliciting DSTs were applied in practice by prospectively collecting data on TB symptoms and MDRTB risk factors in people suspected of having TB (TB suspects). Over a six-week period before implementation of direct MODS DST in the regional laboratory, TB program staff at 45 health clinics were asked to complete a brief (1-2 minutes) data capture sheet for each attending TB suspect. This information was cross-referenced with the TB suspect and laboratory registries at each health clinic.

Before data collection, data capture sheets-an explanation of study purpose and guidance on data recording-were provided to TB program personnel at each clinic. All clinics were contacted two weeks later to identify and address difficulties in data collection.

Data capture sheets were completed when two sputum sample containers were given to the patient and all sheets were collected at the end of the six-week period. After data entry, all clinics were revisited within two weeks to clarify data queries and revise TB suspect and laboratory registries for the period corresponding to data collection.

Existing and projected DST geographic distribution. To estimate the minimum number of DSTs that might be solicited annually within the jurisdiction of the regional laboratory if all eligible samples 
were submitted for direct MODS DST, we used the cross-sectional MDRTB risk factor prevalence data from our prospectively collected data capture sheets and applied them to the annual number of TB suspects attending these centers. Because we had specifically selected sites with a high MDRTB burden, it was not appropriate to extend these data to nonstudy sites where the burden of risk factors is likely lower. Thus, our calculations are underestimates of the total potential workload of the regional laboratory but do indicate the burden from the 45 centers studied (representing 20\% of the health centers in the region), assuming perfect adherence to national DST guidelines. We then compared the annual projected spatial distribution of DST requests by district with the retrospectively traced distribution of 124 DST requests by district in 2005 to determine whether there was evidence of geographic bias.

\section{Ethical review}

Approval for the study was obtained from the Institutional Review Board of Universidad Peruana Cayetano Heredia in Lima, Peru, and the regional Tuberculosis Control Program in Arequipa.

\section{RESULTS}

\section{Laboratory environment}

Laboratory technician time. No additional personnel were provided to facilitate MODS implementation and MODS did not replace the indirect proportions method DST; thus, the new workload was in addition to existing duties for the single laboratory technician.

The TB laboratory technician from Arequipa received four weeks of training in the investigators' TB research laboratory in Lima. Ten days were dedicated to learning other culture methods and to observing MODS, and 10 days were dedicated to participating in processing samples for decontamination, culture, and drug susceptibility in MODS. The technician independently ran more than 40 tests and read more than 240 tests under supervision of laboratory personnel experienced in teaching laboratory technique, who had one to seven years of experience in TB diagnostics.

In the first six weeks when MODS was being implemented, the technician spent
31 hours (13\% of total work time) in MODS-related activities, $77 \%$ of which were dedicated to processing samples. The rest of the technician's MODS-related time was spent troubleshooting implementation problems, in meetings regarding implementation logistics, and reading related materials. In five months, 53.5 $(8 \%)$ of 640 technician work-hours were dedicated to MODS implementation, $92 \%$ (49 hours) of which were dedicated to processing samples. The lab technician processed and read MODS tests on 45 sputum samples during this time.

Overall, processing 20 DSTs required one workday with MODS (from sputum) but four workdays with the proportions method (including strains) in Arequipa where one person performed every step of the procedure, including cleaning and media preparation. The incremental and cumulative time frames of the direct MODS method and the indirect proportions method are compared in Table 1 and Figure 1. Unless otherwise noted, data in Table 1 for the proportions methods are from stopwatch-timed observations in Arequipa alone. MODS data are extrapolated from times for processing 3, 6 , and 12 samples and normalized to 20 for comparison with the proportions method. The estimation of running times

TABLE 1. Estimated time spent per 20 samples processed by microscopic-observation drugsusceptibility (MODS) and proportions methods

\begin{tabular}{lll}
\hline \multicolumn{1}{c}{ Procedure step } & Proportions method & MODS method \\
\hline Washing equipmenta $^{\mathrm{a}}$ & $2-3$ days & 1 hour \\
Culture media preparation $^{\mathrm{b}}$ & 12 hours & 3 hours 30 minutes \\
Antibiotics stocks $_{\text {Autoclaving }}$ & 22 minutes & 16 minutes \\
Decontamination $^{\mathrm{c}}$ & 1 hour & 1 hour \\
Method $_{\text {Reading cultures }}^{\mathrm{d}}$ & 2 hours 39 minutes & 2 hours 39 minutes \\
Recording results $^{\mathrm{e}}$ & 6 hours 48 minutes & 2 hours 25 minutes \\
Total time & 7 hours 5 minutes & 1 hour 40 minutes \\
& 1 hour 20 minutes & 1 hour 20 minutes \\
\hline
\end{tabular}

a Washing equipment was estimated for one washer and is not included in the total time. In addition to pipettes and beakers used for both methods, the proportions method utilizes reusable glass tubes, while MODS utilizes disposable plastic plates. ${ }^{b}$ MODS is performed directly from sputum, while the proportions method is performed from strains. Proportions method media preparation time includes Ogawa media used for culture from sputum as well as for Lowenstein-Jensen, which is used for drug susceptibility testing (DST). MODS media preparation time was estimated by multiple lab technicians and is the same for 1 or 45 samples.

${ }^{c}$ The decontamination process is identical for both proportions and MODS methods.

${ }^{d}$ Reading proportions method takes 5 minutes per sample if sensitive, 15 minutes per sample if resistant. Each proportions method test consists of reading 16 Lowenstein-Jensen agar plates at 21 and 42 days. For the initial Ogawa culture, each reading takes about 38 seconds, and cultures here are read on two occasions. The above calculation assumes the most timeconsuming possibility: culture negativity until final reading of Ogawa and a sensitive DST until the second reading. Reading MODS takes 1 minute per sample and here is assumed as being read at 5, 7, 10,15, and 21 days. While MODS culture positivity is reached at a median of 7 days, the above calculation assumes the most time-consuming possibility: culture negativity until 21 days.

${ }^{e}$ Recording results are similar for both methods and were estimated by multiple lab technicians.

FIGURE 1 Cumulative processing time: proportions method versus microscopic-observation drug-susceptibility (MODS) method

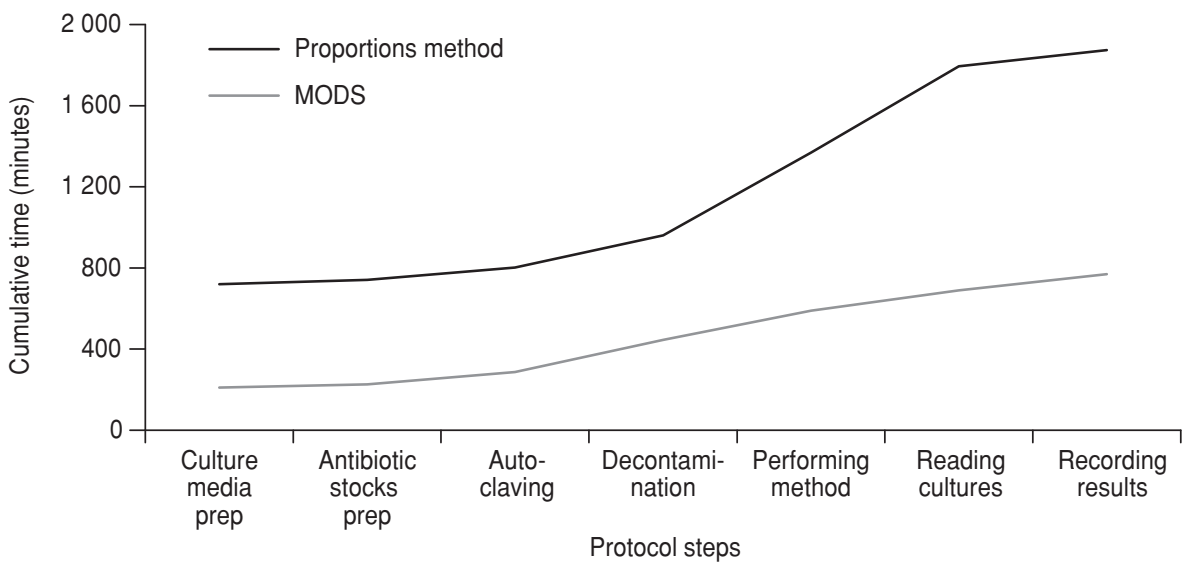


TABLE 2. Itemization: added costs of microscopic-observation drug-susceptibility method

\begin{tabular}{lr}
\hline \multicolumn{1}{c}{ Equipment } & Cost $^{\mathrm{a}}$ \\
\hline Inverted light microscope $^{\mathrm{b}}$ & 2000 \\
Vortex $^{\mathrm{b}}$ & 249 \\
Gilson pipettes (2) & 160 \\
Total cost & 2409 \\
\hline
\end{tabular}

a In U.S. dollars.

b Used equipment.

is limited by economy of scale (and the difficulty of accounting for laboratory multitasking). Thus, incremental and cumulative timing calculations are likely longer than in practice and not externally valid. Note that increments in Figure 1 correspond to Table 1. Cumulative processing times are for 20 samples, and proportions method timings include primary isolation in Ogawa culture.

Capital and recurrent costs. The total added cost of implementing MODS was U.S. \$2 409 (itemized in Table 2). While the consumable unit cost of a MODS test is less than U.S. \$2, the value of the donated initial materials (for 100 tests) in which there was a surplus of many materials was U.S. $\$ 745$. The opportunity cost for training the laboratory technician was U.S. \$455. On the basis of the time dedicated to MODS implementa-

TABLE 3. Metric size of microscopic-observation drug-susceptibility equipment

\begin{tabular}{lcccc}
\hline \multicolumn{1}{c}{ Equipment item } & Height $(\mathrm{m})$ & Width $(\mathrm{m})$ & Depth $(\mathrm{m})$ & Footprint $\left(\mathrm{m}^{2}\right)^{\mathrm{a}}$ \\
\hline Refrigerator & 1.53 & 0.71 & 0.71 & 1.01 \\
Vortex & 0.18 & 0.12 & 0.16 & 0.02 \\
Centrifuge & 0.4 & 0.45 & 0.45 & 0.20 \\
Incubator & 1.62 & 1.19 & 0.75 & 0.89 \\
Autoclave & 0.85 & 0.6 & 0.42 & 0.25 \\
Inverted light microscope & 0.46 & 0.31 & 0.44 & 0.14 \\
Biological safety cabinet & 2.22 & 2.0 & 0.78 & 1.56 \\
\hline
\end{tabular}

a The footprint includes space for opening doors.

tion across five months, the opportunity cost for labor was U.S. \$150 for the pilot period.

Space. Total floor space used for MODS equipment was 3.56 square meters (excluding space for personnel movement). The laboratory in Arequipa measured $3.8 \times 4.8 \times 3.0$ meters and the media preparation room was $5.2 \times 4.3 \times 3.0$ meters, which allowed ample room for multiple personnel. Equipment sizes and footprints are itemized in Table 3.

\section{Existing DST system}

Figure 2 illustrates the stepwise breakdown of the existing DST process, from sputum collection from patients through second-line DST to receipt of results against the projected time frames for direct DST with MODS.
From January 2005 to February 2006, 139 DSTs were done at the regional reference laboratory. The sequential time steps in sample and result delivery were traced as far as possible. Record quality was variable and some time point data were therefore incomplete.

The requesting clinic successfully identified $79(57 \%)$ of the DSTs, even though one-third of the time $(n=23)$ regional reference laboratory paperwork identified the wrong clinic (some were the clinics where the intermediate laboratory was located). Of the 60 untraceable DSTs, 26 had incorrect information listed in regional and subregional laboratories.

Receipt of results in the local clinics was confirmed for one-third $(n=23)$ of the 79 traced DST requests, 9 of which reported MDRTB. Results were received a median of 26 days after being recorded in the regional reference laboratory. In

FIGURE 2. Existing drug susceptibility testing timeline ${ }^{a}$

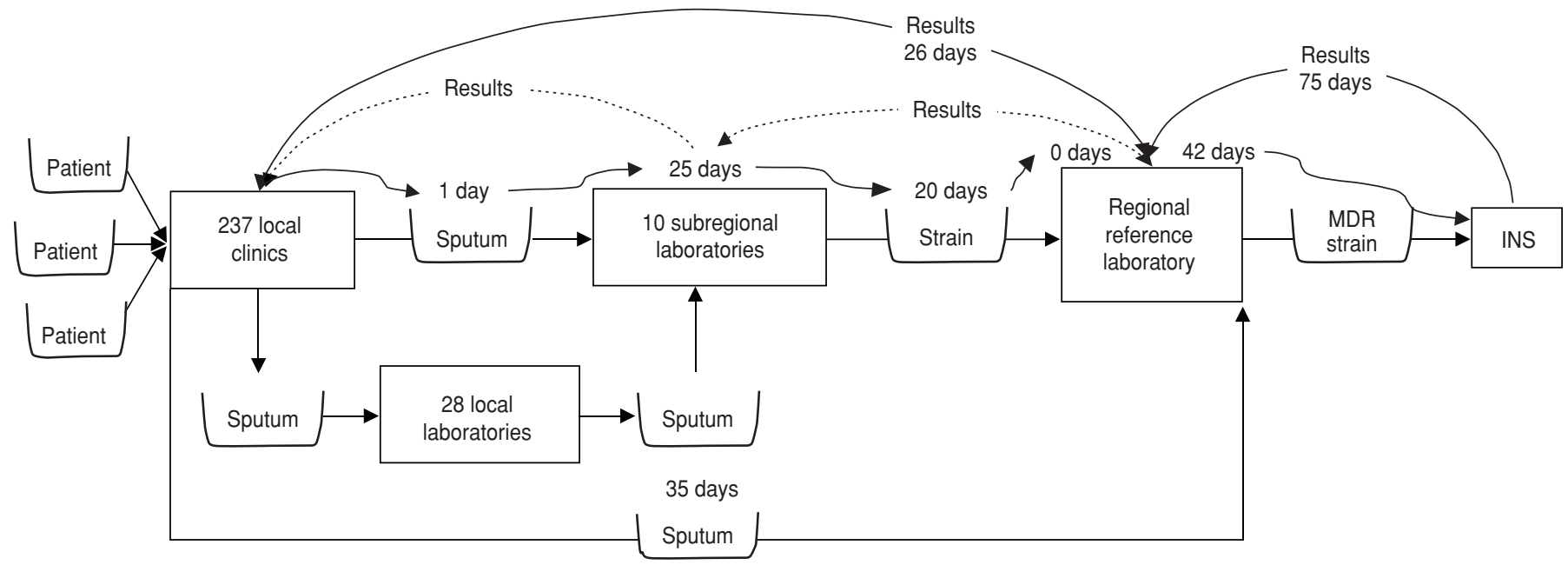

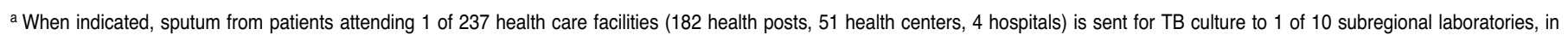
many cases after smear microscopy at 1 of 28 additional local laboratories. If the culture is positive, and if the patient has a risk factor for MDRTB, the strain is sent to the regional reference laboratory for first-line DST. If the strain is MDR, it will be sent on to the Instituto Nacional de Salud (INS) for MDR confirmation and second-line DST. Hard copies of DST results are provided to laboratory and local clinic staff, and results that would change treatment plans should be immediately communicated by telephone. The median delay for the current, indirect DST algorithm from a culture request in a local clinic to DST result at the regional reference laboratory is $1+25+20+0+42=88$ days. It took an average of 26 days for available DST results to be communicated to local clinics ( $n=23$, range 1-370), and the average delay to receipt in the regional laboratory in Arequipa of second-line DST results from the national (INS) reference laboratory was 75 days (range 48-113; based on complete data for 10 of the 23 strains sent). The maximum projected delay to result availability if isolation of strain from sputum was not required and samples were sent directly to the regional reference laboratory is 35 days. 
one case, results had been communicated by telephone before the paperwork was received. The median time from DST request to MDRTB diagnosis and communication of results was just over 16 weeks. The median turnaround time of second-line DST results from the INS to the regional reference laboratory was 2.5 months.

\section{Fidelity to national DST guidelines}

Data on the frequency of MDRTB risk factors in TB suspects and corresponding DST requests were recorded at 45 clinics for an average of 29 days (1 121 clinic days). To reduce sampling bias, centers returning data on fewer than $30 \%$ of registered patients were excluded from the analysis $(n=5)$. These data and those excluded for duplication or patient ineligibility (receiving $\mathrm{TB}$ treatment), accounted for 93 of the 803 data sheets collected. We did not exclude 62 completed data capture sheets lacking a corresponding entry in the clinic registry, likely reflecting patients failing to return a sputum sample (see Figure 3). Thus, data were analyzed for 710 patients, representing $84.0 \%$ of TB suspects recorded in clinic registers during the study period (range in individual clinics $50 \%$ to $150 \%$; some clinics filled data capture sheets for patients not recorded in clinic registers).

According to TB program criteria, $89.2 \%$ of patients $(n=633)$ for whom samples were sent for TB diagnosis fulfilled the definition of a TB suspect. At least one risk factor for MDRTB was recorded in $17.5 \%(n=111)$ of these 633 patients, of whom $18.0 \%(n=20)$ had more than one risk factor. The most common risk factors were prior $\mathrm{TB}$ treatment, oral or inhaled corticosteroid use, and occupational exposure (Figure 4).

According to national TB program guidelines all 111 patients with MDRTB risk factors should have undergone culture and, if culture positive, subsequent DST, but only $10.8 \%$ of these 111 patients $(n=12)$ had a sample sent for culture (Figure 5).

\section{DST request burden versus MDRTB risk factor prevalence mismatch}

To compare the actual geographic distribution of DST requests with that predicted by the prevalence of MDRTB risk factors in TB suspects in our sample of 45

FIGURE 3. Flowchart of attrition and exclusion for evaluation of multidrug-resistant tuberculosis (MDRTB) risk factors in TB suspects, Arequipa, Peru, 6-week period, 2006

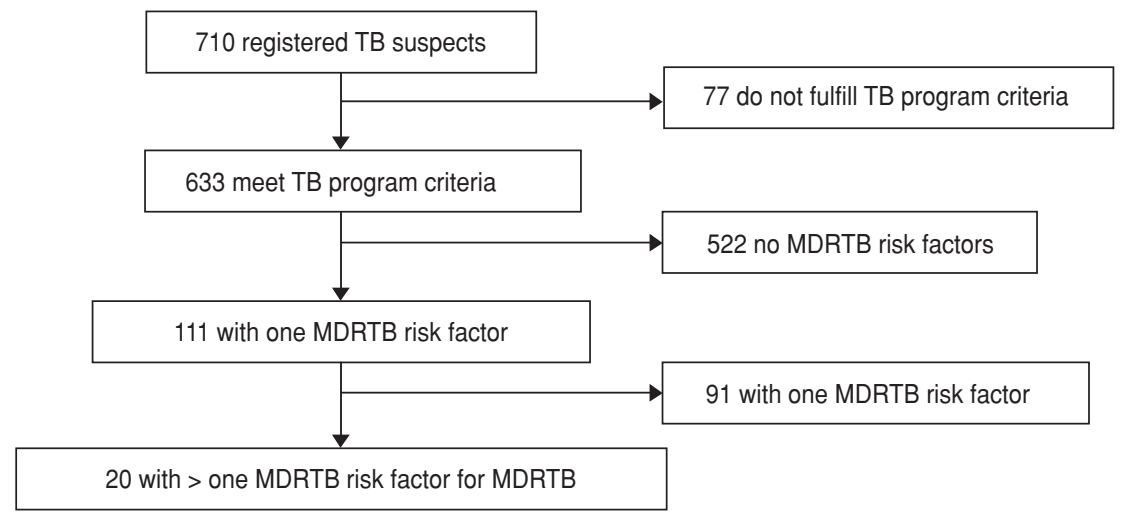

FIGURE 4. Multidrug-resistant tuberculosis (MDRTB) risk factor distribution, Arequipa, Peru, 6-week period, 2006

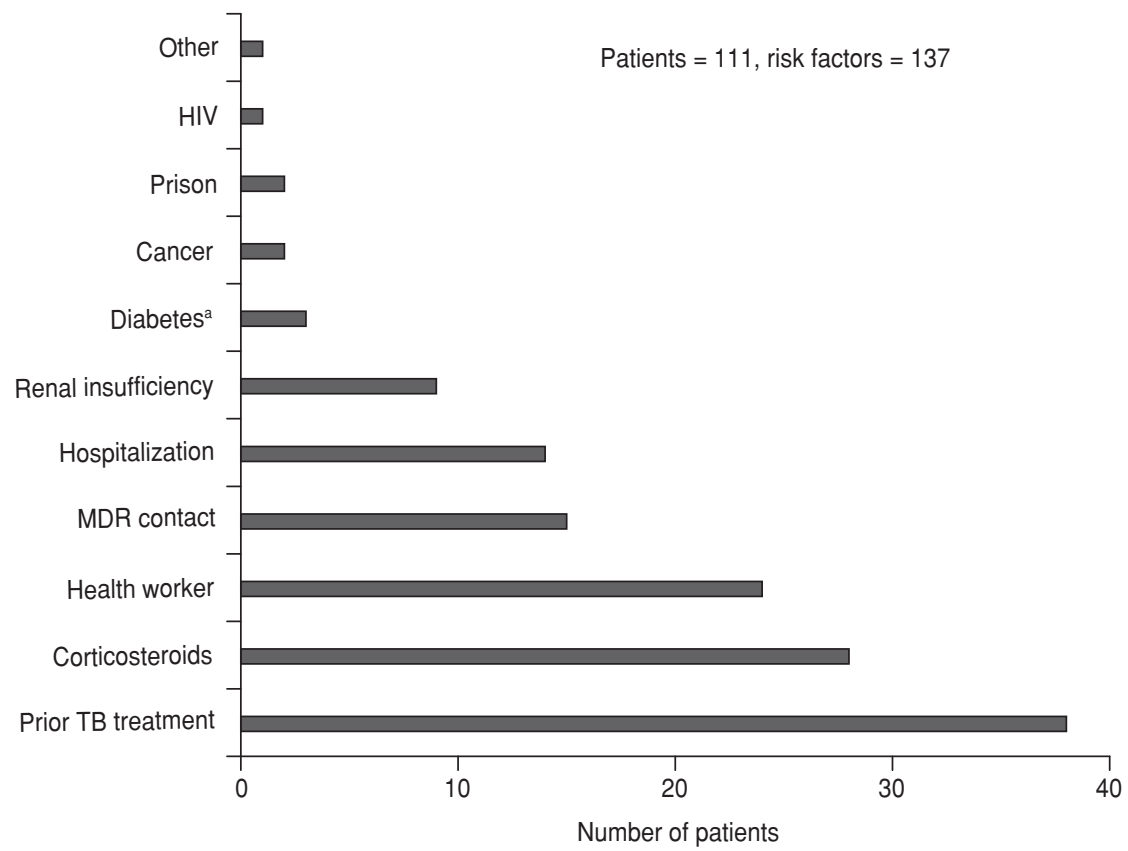

a Diabetes is included as an indication for drug susceptibility testing because of the reported poorer outcomes for diabetic patients, rather than because it is a risk factor for MDRTB per se.

clinics, data on the 124 DSTs performed between January and December 2005 were analyzed.

Figure 6 illustrates the mismatch of the two frequency distributions and most notably an underrepresentation of DST requests from District 5, the district most distant by paved road from the regional reference laboratory (173 kilometers).

\section{DISCUSSION}

Three key findings emerged from this evaluation of the pilot rollout in Peru of a new, rapid diagnostic test for MDRTB.
First, the implementation of a new test parallel to, rather than as a replacement for, existing methods creates demands on funds, personnel, sample transport, and information systems that must be considered alongside new test startup costs. Second, direct DST at the regional reference laboratory avoids primary culture at subregional laboratories and could obviate important delays in sample and result delivery. Third, despite well-defined national guidelines for DST and programs of ongoing in-service training, field application is geographically variable; although one-fifth of $\mathrm{TB}$ 
FIGURE 5. Prospectively recorded diagnostic workup of tuberculosis suspects with multidrug-resistant tuberculosis (MDRTB) risk factors, Arequipa, Peru, 6-week period, 2006

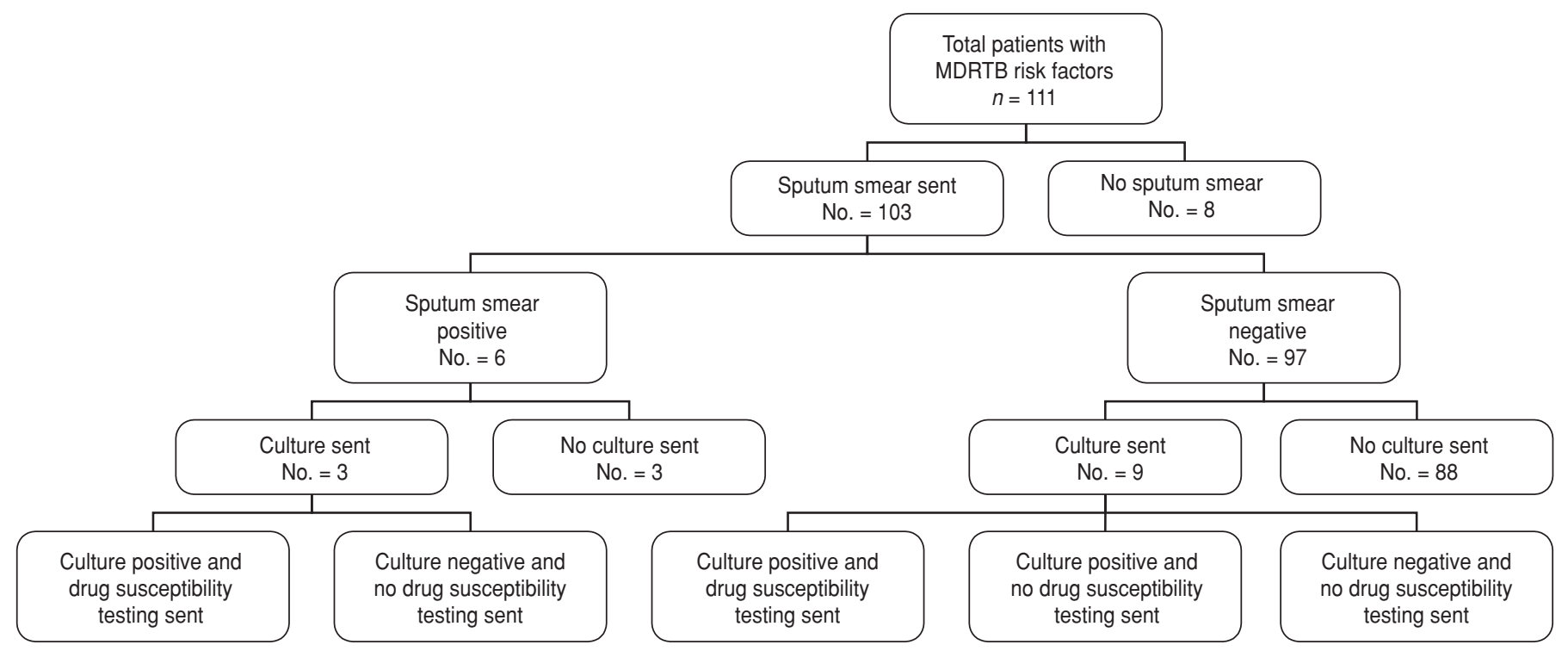

FIGURE 6. Mismatch of drug susceptibility testing (DST) request burden and multidrug-resistant tuberculosis (MDRTB) risk factor prevalence, Arequipa, Peru, 2005

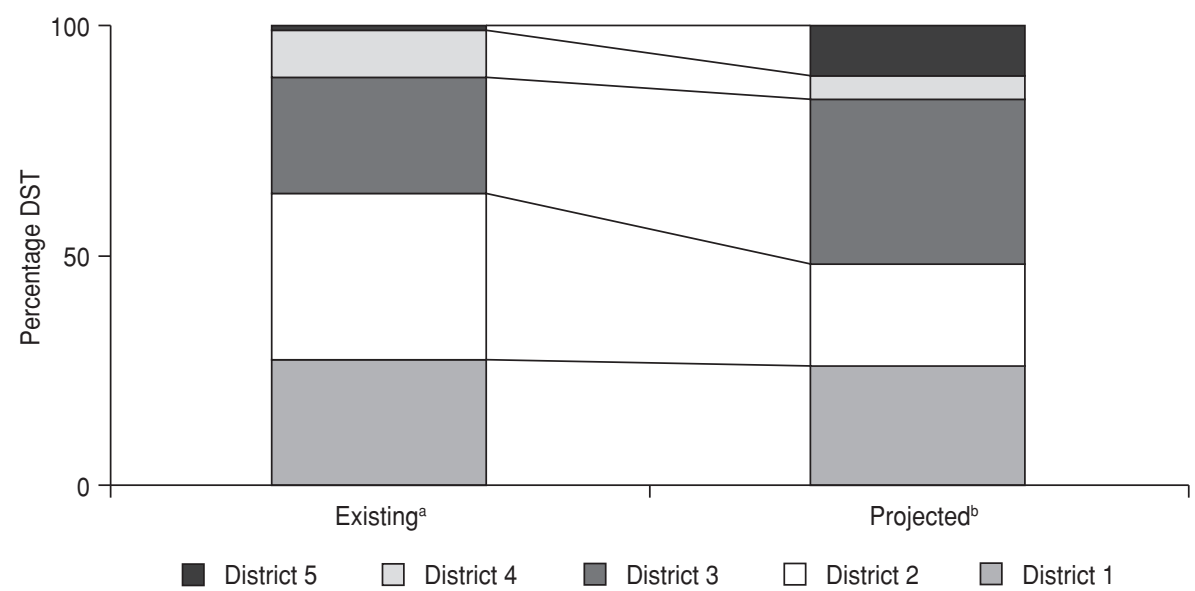

${ }^{a}$ Existing DST data are percentages derived from distribution of requests for the 124 DSTs performed in 2005 (not restricted to the 45 selected high-burden clinics). Five of the six districts in Arequipa were included in the analysis. The excluded district was geographically distant and had a low annual incidence of TB (34.5 per 100 000). None of the DSTs in 2005 came from District 5 , though 724 requests $(20.0 \%$ of the annual 3619 TB suspects in the region) would be projected on the basis of our risk factor data. The proportion of sampled TB suspects presenting with MDRTB risk factors was applied to the total annual number of TB suspects by district recorded by the TB program.

${ }^{b}$ Projected refers to the proportion of DST request burden that would be expected based on MDRTB risk factor prevalence.

suspects fulfilled criteria for DST testing, cultures were sent in only $10.8 \%$ of those warranting such testing.

Our findings build upon those of a previous study in Lima (4) and show that, in order to harness the considerable microbiological advantages of rapid, direct DST, investment must be made to ensure matching improvements in the flow of samples and results and the application of national guidelines in local practice. In addition, streamlining the DST system would likely reduce recordkeeping inconsistencies (detected here for one-third of all DST requests) arising from multiple steps of information transfer and would require reallocating sample transport mechanisms.

Study data collection was hampered by weak information systems, which in turn represent a threat to the field utility of new laboratory technologies. Widen- ing access to local Internet providers and cell phone networks suggests that the use of text messaging, e-mail, and Webbased result delivery might offer a future solution. Innovation and investment in strengthening information systems should be an integral element of implementing diagnostics in resource-limited settings, which brings the added value of use by other disease control programs. Otherwise, much of the technical benefit of rapid direct DST is negated.

In this setting, implementation depended on dynamic support from both the investigators and the TB program. Political will, particularly commitment to sustained funding, as noted in the World Health Organization strategy to Stop TB, is crucial. The initial phase of implementation of direct DST will usually be alongside existing indirect DST with resulting additional material and personnel costs; however, once indirect DST is replaced, existing funding sources should be used to sustain the new method. Thus, the additional commitment required by the TB program is in priming the system with a time frame for phasing out older technology as part of the implementation plan.

A strength of this study was the concurrent multilevel assessment of the DST pathway from peripheral clinics to regional and national reference laboratories. We evaluated the system from the perspective of health workers in the field as well as laboratory technicians at 
the central level. We were able to examine the application of national guidelines in a broad range and number of health clinics. Additionally, the prospective collection of data on TB symptoms and MDRTB risk factors before implementation of MODS ensured that these data were uncolored by the availability of the test. These findings have implications that are generalizable for MODS and other diagnostic tests in similar settings.

We found that MODS can be implemented in a level P2 clinical laboratory without difficulty. Adding capital costs, the value of the donation for consumables for 100 samples, and the opportunity costs of training and labor, implementing MODS in Arequipa cost U.S. $\$ 3759$. While MODS alone has the potential to decrease to nearly one-quarter the time to MDRTB diagnosis in Arequipa, situational analysis identified multiple levels of the health system where intervention should further improve time to MDRTB diagnosis. Matching lab capacity with demand for DST, targeting specific time delays in the existing DST algorithm, and educating health care personnel about national DST guidelines should improve target population access to DST and shorten the overall timeline.

In collecting data on the flow of DST, we were restricted by the variable qual- ity of routinely kept records. A potential source of bias is that data capture sheet completion was inconsistent, and we cannot exclude the possibility that they were more likely to be completed if MDRTB risk factors were noted. We attempted to address this problem by excluding centers with less than $30 \%$ capture of registered TB suspects. Within the study setting, we deliberately selected high MDRTB burden areas and therefore may be overestimating the prevalence of MDRTB risk factors and the predicted burden of DST in the region. Additionally, culture output was initially low, reflecting the difficulty in adding new work to an overburdened system. A dedicated technician can process 100 MODS samples per week (5 000 annually) — training for such a technician is now accomplished in five days, reducing associated opportunity costs.

Our specific objectives were to describe the necessary laboratory environment used for implementation, the existing system for DST, the fidelity of clinical practice in the field to the national recommendations for DST, and the degree to which geographic variation in DST requesting patterns matched that predicted by the geographic variation in reported risk factor prevalence.

The implementation of a new MDRTB diagnostic test in a resource-limited setting presents challenges at the level of the laboratory environment, existing health care system DST procedures, and application of national guidelines in peripheral health clinics. Assessing each of these elements can maximize efficient rollout and utilization of a new tool. These data demonstrate that a facilitating laboratory environment for MODS implementation requires additional personnel time (until MODS replaces the conventional test) and modest financial investment in existing laboratories; the current DST algorithm is labor intensive, too slow to deliver results within a clinically meaningful time frame and wastefully ineffective, with only one-third of results reaching the requesting clinician; disenchantment with this service has led to a virtual abandonment of the national guideline criteria for DST requesting in peripheral health care facilities; however, if fidelity to the national guidelines were $100 \%$, the potential laboratory workload would be enormous as risk factors are extremely common. Key to improving the service delivery of the diagnostic TB laboratory is in strengthening systems for transferring samples to the laboratory and delivering results to the requesting clinic; furthermore, investment in personnel and laboratory resources is integral to harnessing the benefits that high-performance new diagnostic tests offer and can bring added value to other programs within the health care system.

\section{REFERENCES}

1. Heifets LB, Cangelosi GA. Drug susceptibility testing of Mycobacterium tuberculosis: a neglected problem at the turn of the century. Int J Tuberc Lung Dis. 1999;3(7):564-81.

2. Mukherjee JS, Rich ML, Socci AR, Joseph JK, Viru FA, Shin SS, et al. Programmes and principles in treatment of multidrug-resistant tuberculosis. Lancet. 2004;363(9407):474-81.

3. Walker D. Economic analysis of tuberculosis diagnostic tests in disease control: how can it be modelled and what additional information is needed? Int J Tuberc Lung Dis. 2001;5(12): 1099-108.

4. Yagui M, Perales MT, Asencios L, Vergara L, Suarez C, Yale G, et al. Timely diagnosis of MDR-TB under program conditions: is rapid drug susceptibility testing sufficient? Int J Tuberc Lung Dis. 2006;10(8):838-43.

5. Moore DA, Evans CA, Gilman RH, Caviedes L, Coronel J, Vivar A, et al. Microscopicobservation drug-susceptibility assay for the diagnosis of TB. N Engl J Med. 2006;355(15): 1539-50.

6. Caviedes L, Lee TS, Gilman RH, Sheen P, Spellman E, Lee EH, et al. Rapid, efficient de- tection and drug susceptibility testing of $M y$ cobacterium tuberculosis in sputum by microscopic observation of broth cultures. J Clin Microbiol. 2000;38(3):1203-8.

7. Moore DA, Mendoza D, Gilman RH, Evans CA, Hollm Delgado MG, Guerra J, et al. Microscopic observation drug susceptibility assay, a rapid, reliable diagnostic test for multidrug-resistant tuberculosis suitable for use in resource-poor settings. J Clin Microbiol. 2004;42(10):4432-7.

8. Moore DA, Caviedes L, Gilman RH, Coronel $\mathrm{J}$, Arenas F, LaChira $\mathrm{D}$, et al. Infrequent MODS TB culture cross-contamination in a high-burden resource-poor setting. Diagn Microbiol Infect Dis. 2006;56(1):35-43.

9. Shiferaw G, Woldeamanuel Y, Gebeyehu M, Girmachew F, Demessie D, Lemma E. Evaluation of microscopic observation drug susceptibility assay for detection of multidrugresistant Mycobacterium tuberculosis. J Clin Microbiol. 2007;45(2):1093-107.

10. Arias M, Mello FC, Pavon A, Marsico AG, Alvarado-Galvez C, Rosales S, et al. Clinical evaluation of the microscopic-observation drug-susceptibility assay for detection of tuberculosis. Clin Infect Dis. 2007;44(5):674-80.

11. Solis LA, Shin SS, Han LL, Llanos F, Stowell M, Sloutsky A. Validation of a rapid method for detection of $M$. tuberculosis resistance to isoniazid and rifampin in Lima, Peru. Int J Tuberc Lung Dis. 2005;9(7):760-4.

12. Unidad Técnica de TB Multiresistente. Tasas de incidencia anual (TIA) de TB MDR en mayores de 15 años por departamento del Perú: reporte de MDR. Estrategia Sanitaria Nacional de Prevención y Control de la Tuberculosis. 2006;3:2.

Manuscript received on 2 July 2008. Revised version accepted for publication on 29 January 2009. 
RESUMEN Objetivos. En un proyecto piloto para la implementación de la metodología de observación microscópica para determinar la susceptibilidad a medicamentos se identificaron los retos logísticos y del sistema de salud que se deben atender para aprove-

Preparativos de campo y laboratorio: retos para la introducción de nuevos diagnósticos de la tuberculosis multirresistente char los beneficios de nuevas y prometedoras herramientas diagnósticas para la tuberculosis resistente a múltiples medicamentos (TRMM).

Métodos. Los datos regionales sobre las prácticas y el desempeño del sistema de salud relacionados con los algoritmos de diagnóstico de la TRMM se recogieron en los centros de salud y los laboratorios locales.

Resultados. La implementación paralela de una nueva prueba y el método actual crea demandas de recursos, personal, transportación de las muestras y sistemas de información adicionales a los costos de introducción de la nueva prueba. Eliminar la necesidad de cultivos primarios en laboratorios intermedios mediante la prueba de susceptibilidad a los medicamentos (PSM) directa en el laboratorio regional de referencia reduce significativamente la demora. La aplicación en el terreno de directivas nacionales bien definidas para las PSM es desigual. Si la fidelidad a las directivas nacionales fuera perfecta, la demanda de PSM aumentaría más de 50 veces, con implicaciones importantes para la capacidad de laboratorio.

Conclusiones. La implementación de un nuevo diagnóstico de TRMM presenta retos para el sistema de laboratorio, el proceso actual de PSM y la aplicación de las directivas nacionales en las clínicas periféricas. La evaluación de cada elemento puede optimizar el uso eficiente de una nueva herramienta diagnóstica. En particular, el fortalecimiento de los sistemas de transportación de muestras al laboratorio y el envío de los resultados a las clínicas solicitantes, además de la inversión en personal y recursos de laboratorio, son requisitos para aprovechar los beneficios de las nuevas pruebas diagnósticas de alto rendimiento y pueden brindar valor añadido a otros programas del sistema de atención sanitaria.

Palabras clave Mycobacterium tuberculosis; tuberculosis resistente a múltiples medicamentos; implementación de plan de salud; técnicas de laboratorio clínico; Perú. 